\title{
Who should care for people with bleeding disorders?
}

By Cathy Harrison

An integrated model of specialised-delivered care is widely accepted as the standard of care for people with haemophilia in the UK. Assessment of available evidence on patient outcomes confirms this approach. But leading the specialist care for this group of patients does not require a medical qualification. Specialist nursing is well established within the haemophilia service and offers perhaps the greatest resource as health services cope with cost constraints on the specialist provision of services.

Keywords: Haemophilia, guideline, care model

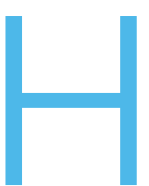

aemophilia is a rare disorder that is complex to diagnose and manage. Optimal care warrants more than treating an acute bleed. Priorities lie in the improvement of health and quality of life of people with haemophilia (PWH), including prevention of bleeding and joint arthropathy, prompt cessation of bleeding, management of diseaseassociated complications such as inhibitors and viruses, and attention to psychosocial health ${ }^{[1]}$. The condition is expensive to treat and its management varies through diverse health services and economic systems worldwide.

The care of PWH is commonly provided by a specialised multidisciplinary team, but this varies significantly throughout the world. In the UK, comprehensive care centres provide 24-hour specialist care for PWH and other bleeding disorders, but this is not the case everywhere as a result of issues including a lack of specialists, products and diagnostic technology. A team from the National Hemophilia Foundation and McMaster University recently published a systematic

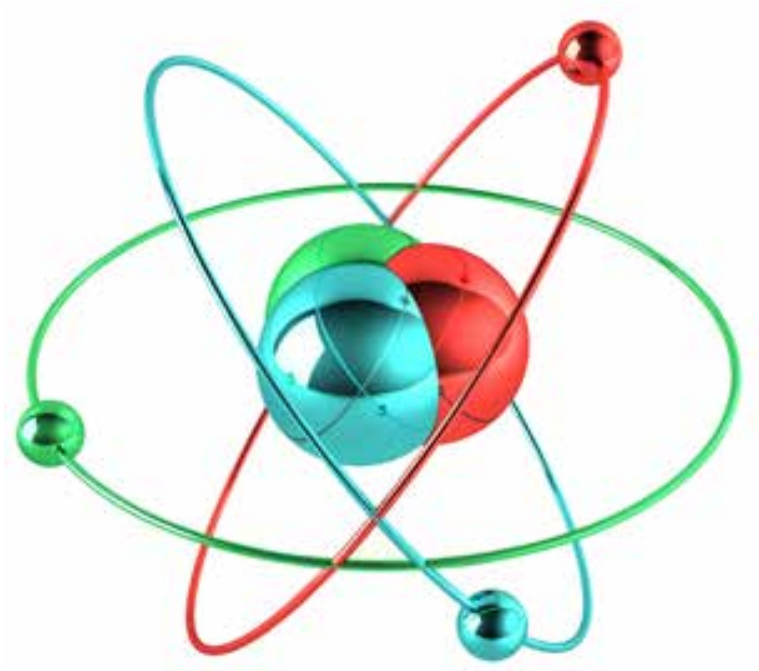

review of published studies assessing the value of the integrated care model on patient outcomes for $\mathrm{PWH}^{[2]}$. The outcomes reviewed were mortality, missed days of school or work, emergency department visits, length of in-patient stay, quality of life, joint damage or disease, educational attainment, patient adherence and patient knowledge. The models of care reviewed were the specialised care model, identified since the 1940s as the preferred model of care in the UK ${ }^{[1]}$; care delivered by a non-specialist in a non-specialist setting and; the 'no care' model, which was assumed not to occur in the Western world.

NHF-McMaster's collaborative systematic review of models of care focused on the recommendations for models of care for PWH living in the United States ${ }^{[2]}$. Did the review tell us anything we did not know, or did not think we already knew? The answer, in short, is no. However, it confirms our current knowledge, ensuring that we are providing evidence-based care. As the UK healthcare system changes in the coming years and commissioning groups require evidence to support what we recognise as 'gold standard' care, this paper will certainly go some way towards providing that. 
The NHF-McMaster team chose to review other patient cohorts alongside PWH due to the rare nature of haemophilia, which they argue enabled them to draw firmer conclusions from the evidence ${ }^{[3]}$. There are no randomised studies in the delivery of care for $\mathrm{PWH}$ or cystic fibrosis and it would be unethical to randomise and compare the care of these individuals between GP or specialist-led care. While the education around such as asthma and diabetes fits well, comparing $\mathrm{PWH}$ with those who have these chronic conditions does not quite fit. Conditions such as rheumatological disorders may provide a better comparator. The difference in numbers and the capacity of primary care to deal with specialist rare conditions seems more comparable. There is evidence to show that the integrated model of care provides improved patient outcomes and satisfaction within rheumatology disorders ${ }^{[4,5,6]}$.

\section{"The development of nurse-led services could ensure that patient care continues to be individualised in the future"}

Data collection is essential in order to manage rare conditions appropriately. Both CF and haemophilia teams have been doing this for decades ${ }^{[7,8,9]}$. Although well controlled studies are required to establish the benefit of new specialist treatments, the value of compiling data from a wider clinical population cannot be ignored. Databases such as EUHASS in haemophilia, for example, provide valuable information on the management of this rare condition.

The NHF-McMaster guideline, while recommending comprehensive care for all, states that the evidence is stronger for those with inhibitors. Does this therefore mean it is acceptable for others not to be managed within a comprehensive care setting? PWH and inhibitors are complex, but if the management of ageing patients with mild haemophilia is not given careful consideration, the development of inhibitors following complex surgery, for example, can be a devastating health outcome. Patient-related, non-modifiable risk factors, as well as environmental modifiable risk factors, have been identified in inhibitor development ${ }^{[11,12]}$.

We are working in a new era of haemophilia care. With $\mathrm{PWH}$ reaching older ages, we are now seeing the emergence of (and managing) conditions commonly seen in the general population, notably cancer and cardiovascular disease. Managing complex surgeries and malignancies clearly requires specialist support ${ }^{[13,14]}$. Evidence is now accumulating on the complexity of managing cardiovascular disease in $\mathrm{PWH}$, but this is not something to be managed in primary care or even by an independent cardiologist ${ }^{[15,16]}$. Even as specialists, we are treading new ground in caring for $\mathrm{PWH}$, and to allow the possibility of primary care, internal medicine, oncology or cardiology medics managing these patients without specialist haemophilia team input is unsafe. The management of comorbidities requires extensive comprehensive care.

I would argue, however, that leading the specialist care for this group of patients does not require a medical qualification. Specialist nursing within the care of PWH is well established, and nursing teams have a more hands-on and constant presence in patients lives than their medical counterparts. As such, they have much more ownership of this service. Nurse consultants have been present within the speciality for 20 years and there are a growing number of advanced nurse practitioner roles working to a higher academic level, performing nurse-led research, and presenting and publishing their findings. Nurses with advanced clinical skills are already plugging the gaps within the service where there is a lack of junior doctors with the necessary knowledge. As fewer junior doctors choose haemophilia as a specialty, the development of nurseled services could ensure that patient care continues to be individualised in the future. While a team approach will remain essential going forward, I would argue the specialist nurse and physiotherapist are the best candidates to support other services.

The future of haemophilia care could be very different. Clinical trials of novel treatments are revealing promising results, and it is likely that in $\mathbf{2 0}$ years a specialist team will only be required for very complex procedures and management. However, I would envisage that there will be an additional need for specialist input during the introduction of novel treatments. Rather than focusing purely on haemophilia, we should also be taking a more active role in managing other conditions such as Glanzmann's thrombasthenia, von Willebrand disease, female carriers and other rare factor deficiencies. The Rare Bleeding Disorder Database collects data on all of these rare haemostasis disorders, none of which could be managed outside specialist units ${ }^{[17]}$.

In conclusion, while the NHF-McMaster paper adds important evidence to the management of this rare condition, it really only just begins to scratch the surface on the important question of who should 
care for people with haemophilia. The complexity of the condition is underestimated, and while the paper recognises the importance of specialist care for those with inhibitors, it leaves the rest of the $\mathrm{PWH}$ cohort open to below-standard care. Additionally, while it looks at the evidence for what we recognise as good standard care, it does not strive to make changes to care. In the UK, we should recognise that our greatest resource is our specialist nursing teams. In order to build evidence for the safe management of less prevalent bleeding disorders, changing specialist provision of services, the ageing population of $\mathrm{PWH}$ and novel treatments, it is essential that data collection continues internationally.

\section{Disclosures}

The author has advised no interests that might be perceived as posing a conflict or bias.

This is an Open Access article distributed under the terms of the Creative Commons Attribution License (http://creativecommons.org/licenses/by/2.0), which permits unrestricted use, distribution, and reproduction in any medium, provided the original work is properly cited.

\section{References}

1. Srivastava A, Brewer AK, Mauser-Bunschoten EP, et al. Guidelines for the management of hemophilia. Haemophilia 2013; 19(1): e1-47.

2. Pai M, Key NS, Skinner M, et al. NHF-McMaster quideline on care models for haemophilia management. Haemophilia 2016; 22 (Suppl. 3): 6-16

3. Yeung C, Santesso N, Zeraatkar D, et al. Integrated multidisciplinary care for the management of chronic conditions in adults: an overview of reviews and an example of using indirect evidence to inform clinical practice recommendations in the field of rare disease. Haemophilia 2016; 22 (Suppl. 3): 41-50.
4. Kaupp MJ, Säilä H, Belt EA, Hakala M. Beware of the biologicals hospitals may die: the Rheumatism Foundation Hospital, Heinola Finland (1951-2010). Clin Rheumatol 2012; 31(8): 1151-4.

5. The King's Fund. Haywood Rheumatology Centre - case study. October 2014. Available from https://www.kingsfund. org.uk/sites/files/kf/media/haywood-rheumatology-centrekingsfund-oct14.pdf (accessed 14 November 2016).

6. Väre P. Nikophorou E, Hannonen P, Sokka T. Delivering a one-stop, integrated, and patient-centred service for patients with rheumatic diseases. SAGE Open Med 2016; 14:4. doi: 10.1177/2050312116654404.

7. Corey M, McLaughlin FJ, Williams M, Levison H. A comparison of survival, growth and function in patients with cystic fibrosis in Boston and Toronto. J Clin Epid 1988; 41(6): 583-91.

8. Cystic Fibrosis Foundation. Cystic Fibrosis Foundation Patient Registry 2011 Annual Data Report. Bethesda, Maryland: Cystic Fibrosis Foundation, 2012. Available from http://www.cysticfibrosisdata.org/ReportsUS.html (accessed 14 November 2016).

9. Hay C. The UK haemophilia database: a tool for research audit and healthcare planning. Haemophilia 2004; 10: 21-25.

10. Makris M, Calizzani G, Fischer K, et al. EUHASS: The European Haemophilia Safety Surveillance system. Thromb Res 2011; 127 (Suppl. 2): 22-25.

11. Astermark J. Inhibitor development: patient-determined risk factors. Haemophilia 2010; 16(102): 66-70.

12. Chambost $\mathrm{H}$. Assessing risk factors prevention of inhibitors in haemophilia. Haemophilia 2010; 16 (Suppl. 2): 10-15.

13. Franchini $M$, Tagliaferri A, Mannucci PM. The management of haemophilia in elderly patients. Clin Interv Aging 2007; 2(3): 361-8.

14. Franchini M, Mannucci PM. Past, present and future of hemophilia: a narrative review. Orphanet J Rare Dis 2012; 7:24.

15. Mannucci PM, Mauser-Bunschoten EP. Cardiovascular disease in haemophilia patients: a contemporary issue. Haemophilia 2010; 16 (Suppl. 3): 58-66.

16. Coppola A, Tagliaferri A, Franchini M. The management of cardiovascular diseases in patients with hemophilia. Semin Thromb Hemost 2010; 36: 91-102.

17. Peyvandi F, Palla R, Menegatti M, et al. Coagulation factor activity and clinical bleeding severity in rare bleeding disorders: results from the European Network of Rare Bleeding Disorders. J Thromb Haemost 2012; 10(4): 615-21.

\section{The Journal of Haemophilia Practice}

\section{An open-access journal for sharing experience in the care of people with bleeding disorders}

www.haemjournal.com 